\title{
Real world data on follicular lymphoma patients treated by rituximab-containing immunochemo- therapy and rituximab maintenance
}

\author{
Hee Kyung Kim ${ }^{1}$, Wonseok Kang ${ }^{2}$, Dong Hyun Sinn², Joon Hyeok Lee², Won Seog Kim³ and Seok Jin Kim³
}

\begin{abstract}
${ }^{1}$ Department of Internal Medicine, Chungbuk National University Hospital, Cheongju; Divisions of ${ }^{2}$ Gastroenterology and ${ }^{3}$ Hematology-Oncology, Department of Medicine, Samsung Medical Center, Sungkyunkwan University School of Medicine, Seoul; ${ }^{4}$ Department of Health Sciences and Technology, Samsung Advanced Institute of Health Sciences and Technology (SAIHST), Sungkyunkwan University, Seoul, Korea
\end{abstract}

Received: May 29, 2018 Revised : July 4, 2018 Accepted: July 10, 2018

\section{Correspondence to}

Seok Jin Kim, M.D.

Division of Hematology-Oncology, Department of Medicine, Samsung Medical Center, Sungkyunkwan University School of Medicine, 81 Irwon-ro, Gangnam-gu, Seoul o6351, Korea Tel: $+82-2-3410-1766$

Fax: +82-2-3410-1754

E-mail: kstwoh@skku.edu
Background/Aims: Real-world data about the treatment outcomes of patients receiving rituximab-containing immunochemotherapy followed by rituximab maintenance are required to understand better the treatment for follicular lymphoma (FL).

Methods: A cross-sectional study analyzed FL patients who were treated with R-CVP (rituximab, cyclophosphamide, vincristine, and prednisone) or R-CHOP (rituximab, cyclophosphamide, doxorubicin, vincristine, and prednisone) and rituximab maintenance.

Results: Of 139 patients, 85 patients received R-CVP and 54 received R-CHOP. The characteristics did not differ significantly between the groups. Only grade 3 of FL was more common in R-CHOP. The complete response rate did not differ significantly between R-CHOP (50/54, 92.6\%) and R-CVP (77/85, 90.6\%). The number of disease relapses during rituximab maintenance did not differ significantly between the groups $(p=0.798)$. Therefore, the comparison of progression-free survival (PFS) showed no significant difference: the 3-year PFS rates for R-CVP and R-CHOP were $77 \%$ and $85 \%$, respectively $(p=0.567)$. Although five of 56 hepatitis B virus (HBV) core antibody (anti-HBc)-positive patients experienced HBV reactivation, all cases of $\mathrm{HBV}$ reactivation were identified during regular monitoring for HBV DNA in blood, and were successfully managed with antiviral treatment.

Conclusions: The survival outcomes of FL patients on rituximab maintenance after responding to R-CVP or R-CHOP were similar. Rituximab-containing immunochemotherapy followed by rituximab maintenance can be safely used for anti-HBc-positive patients if HBV DNA titer in blood can be regularly monitored.

Keywords: Lymphoma, follicula; Rituximab; Hepatitis B; Immunochemotherapy

\section{INTRODUCTION}

Follicular lymphoma (FL) is one of the most common subtypes of non-Hodgkin lymphoma (NHL), and its incidence is higher in Western countries than in Asian countries $[1,2]$. In Korea, FL accounts for $5 \%$ of B-cell
NHL, and has clinical characteristics comparable to those seen in Western countries [3,4]. The treatment outcomes of FL patients have markedly improved since the introduction of rituximab, an anti-CD2o monoclonal antibody [5]. Thus, for patients with advanced-stage FL and high tumor burden, rituximab-containing im- 
munochemotherapy is considered the standard of care [6]. However, it is still not clear which immunochemotherapy regimen is the best for FL with a high tumor burden. Thus, some physicians prefer to use R-CVP (rituximab, cyclophosphamide, vincristine, and prednisone) for patients with grades 1 to 3 a whereas others prefer R-CHOP (rituximab, cyclophosphamide, doxorubicin, vincristine, and prednisone) for patients regardless of histologic grade. Recently, the long-term results of the FOLLO5 study showed that patients initially treated with R-CVP had a higher risk of lymphoma progression compared with those receiving R-CHOP [7]. However, the study failed to show better overall survival (OS) after R-CHOP compared with R-CVP. Maintenance therapy with rituximab is similarly debated because it was reported to prolong progression-free survival (PFS) but not OS [8]. Thus, the use of rituximab maintenance as a standard treatment for FL could be controversial, given its high cost and related toxicity. In addition, hepatitis $\mathrm{B}$ virus (HBV) reactivation is frequent in lymphoma patients who are treated with rituximab-containing immunochemotherapy $[9,10]$. Rituximab definitely has a risk of $\mathrm{HBV}$ reactivation in patients positive for hepatitis B surface antigen (HBsAg) [11,12]. However, rituximab may also induce HBV reactivation in HBsAg-negative but HBV core antibody (anti-HBc)-positive patients, because the presence of anti-HBc antibody reflects prior exposure to HBV including occult HBV infection $[13,14]$. Thus, R-CVP and R-CHOP treatment have been found to significantly increase the incidence of HBV reactivation in anti-HBc-positive patients $[15,16]$. However, there are few studies on the impact of rituximab maintenance on $\mathrm{HBV}$ reactivation in HBsAg-negative and anti-HBc-positive patients with FL.

Although previous studies have compared the outcomes of rituximab-containing immunochemotherapy and rituximab maintenance, most data were from clinical trials $[6,17]$. Thus, real-world data reflecting the clinical aspects of FL patients treated with rituximab-containing immunochemotherapy followed by rituximab maintenance might be helpful to understand this treatment approach better. However, such data are limited [18]. Therefore, we performed a study comparing FL patients who were treated with R-CVP or R-CHOP and rituximab maintenance therapy in clinical practice.

\section{METHODS}

\section{Patients}

This was a cross-sectional study analyzing FL patients who were treated with R-CVP or R-CHOP and rituximab maintenance. The study participants met the following criteria. First, they had completed the planned number of cycles of R-CVP or R-CHOP after diagnosis. Any patient whose disease progressed during R-CVP or $\mathrm{R}-\mathrm{CHOP}$ was excluded from the analysis. Second, patients had received at least one cycle of rituximab maintenance therapy after their complete or partial response to R-CVP or R-CHOP was confirmed. Thus, at the time of analysis, patients could have completed the planned 12 cycles of rituximab maintenance or could be undergoing maintenance therapy at the time of analysis. The chemotherapy schedules for R-CVP and R-CHOP are as follows: R-CVP (day 1: rituximab $375 \mathrm{mg} / \mathrm{m}^{2}$ intravenous [IV], cyclophosphamide $1,000 \mathrm{mg} / \mathrm{m}^{2} \mathrm{IV}$, vincristine 1.4 $\mathrm{mg} / \mathrm{m}^{2} \mathrm{IV}$ [maximum $2 \mathrm{mg}$ ]; and days 1 to 5 , prednisone $100 \mathrm{mg}$ orally [PO]); R-CHOP (day 1: rituximab $375 \mathrm{mg} / \mathrm{m}^{2}$ IV, cyclophosphamide $750 \mathrm{mg} / \mathrm{m}^{2} \mathrm{IV}$, doxorubicin 50 $\mathrm{mg} / \mathrm{m}^{2} \mathrm{IV}$, vincristine $1.4 \mathrm{mg} / \mathrm{m}^{2} \mathrm{IV}$ [maximum $2 \mathrm{mg}$ ]; and days 1 to 5, prednisone $100 \mathrm{mg}$ PO). The interim response evaluation was performed after the completion of the third cycle of R-CVP or R-CHOP chemotherapy, and the final response was evaluated after the patients' last cycle of chemotherapy, usually the sixth cycle. Only patients who achieved a complete or partial response to R-CVP or R-CHOP underwent rituximab maintenance. Rituximab ( $375 \mathrm{mg} / \mathrm{m}^{2}$ IV injection per cycle) treatment was repeated every 2 months for 2 years (maximum 12 cycles). A surveillance computed tomography (CT) scan was regularly performed every three to four cycles of rituximab maintenance to check for disease relapse. If a patient showed symptoms or signs suspicious of disease relapse, the CT scan was performed earlier than the planned schedule.

\section{HBV evaluation and monitoring}

Given the risk of rituximab-induced HBV reactivation, serologic markers for $\mathrm{HBV}$ status including $\mathrm{HBsAg}$, hepatitis B surface antibody (anti-HBs), and anti-HBc antibody were evaluated in all patients at diagnosis before initiating rituximab-containing chemotherapy. Serum HBsAg, anti-HBc, and anti-HBs were measured 
according to the manufacturer's instructions, and serum HBV DNA level was measured using the COBAS TaqMan HBV quantitative test (Roche Molecular Systems Inc., Branchburg, NJ, USA). The lower limit of detection for HBV DNA was $9 \mathrm{IU} / \mathrm{mL}$. Patients who were positive for HBsAg received continuous antiviral prophylaxis with entecavir or tenofovir from the commencement of the R-CVP or R-CHOP induction chemotherapy. In patients who were positive for immunoglobulin $\mathrm{G}$ anti-HBc antibody, we measured the level of HBV DNA in blood to confirm the absence of HBV before commencing rituximab-containing chemotherapy. Liver function tests (LFTs) including liver enzymes were performed for all patients whenever they visited the outpatient clinic, regardless of their symptoms or rituximab administration. In addition, HBV DNA in blood was measured every three to four months during induction and rituximab maintenance for patients who were positive for anti-HBc antibody.

\section{Study objectives and analysis}

The primary objective was to compare the PFS of patients who received R-CVP and rituximab maintenance with that of patients who received R-CHOP followed by rituximab maintenance. The secondary objectives included a comparison of the OS, frequency of HBV reactivation, and mortality in the two groups. HBV reactivation was defined as the occurrence of symptomatic hepatitis in a previous healthy HBV carrier who was positive for HBsAg with normal LFT before immunochemotherapy. In patients who were positive for anti-HBc without $\mathrm{HBsAg}, \mathrm{HBV}$ reactivation was defined as an increase in HBV DNA in blood by $\geq 10 \mathrm{IU} / \mathrm{mL}$ or seroconversion to HBsAg positive [19]. In addition, we performed risk factor analysis for survival outcome to explore potential risk factors affecting the survival outcomes of R-CVP or R-CHOP followed by rituximab maintenance. We selected 139 patients who fulfilled the aforementioned criteria from our lymphoma registry between July 2005 and December 2017, after we excluded patients who failed to respond to induction chemotherapy. Because rituximab maintenance therapy has been reimbursed by health insurance in Korea since 2012, all but two of the included patients were diagnosed with FL between 2011 and 2017. All clinical and laboratory data were collected by review of medical records, and the Fol- licular Lymphoma International Prognostic Index (FLIPI) comprising age $\geq 60$ years, Ann Arbor stage III or IV, hemoglobin $<12 \mathrm{~g} / \mathrm{dL}$, elevated levels of serum lactate dehydrogenase ( $\mathrm{LDH})$, and $\geq 5$ involved nodes was used as a prognostic model [20]. The most recent update of disease and survival status was done on April 30, 2018. The final response to R-CVP or R-CHOP induction chemotherapy was reassessed by the investigators according to the Lugano response criteria for NHL [21]. Thus, a score of 1 to 3 for lymph nodes or extralymphatic sites on a 5-point positron emission tomography (PET)-CT scale after the completion of R-CVP or R-CHOP was designated as a complete response whereas the presence of any lesion with a score of 4 or 5 with reduced uptake compared with the PET-CT findings at baseline was designated as a partial response. This study was approved by the Institutional Review Board of Samsung Medical Center, Seoul, Korea, and the requirement for informed consent was waived because of the retrospective nature of the study (No. 2017-02-021).

\section{Statistical analysis}

Differences in baseline characteristics between patients receiving R-CVP and R-CHOP induction chemotherapy were analyzed using a chi-square test. The Kaplan-Meier method was used for the univariate analysis of survival outcomes, and a log-rank test was used for comparisons. PFS was measured from the date of the first day of the first cycle of R-CVP or R-CHOP to the date of death from any cause or the date of disease progression or relapse. OS was measured from the same date as PFS to the date of death from any cause, and was censored at the date of the last follow-up visit. For the analysis of HBV reactivation, events were defined as the occurrence of HBV reactivation at any time until the last follow-up visit or death. Cumulative incidence was calculated by Kaplan-Meier analysis with patients censored on the day of death or last follow-up. Statistical associations were determined by the log-rank test. Two-sided $p$ values $<0.05$ were considered significant. All analyses were performed using SPSS version 23.0 (IBM SPSS Inc., Armonk, NY, USA). 
Table 1. Patient characteristics

\begin{tabular}{|c|c|c|c|c|}
\hline Characteristic & $\begin{array}{c}\text { Total } \\
(\mathrm{n}=139)\end{array}$ & $\begin{array}{c}\text { R-CHOP } \\
(\mathrm{n}=54)\end{array}$ & $\begin{array}{l}\text { R-CVP } \\
(\mathrm{n}=85)\end{array}$ & $p$ value \\
\hline \multicolumn{5}{|l|}{ Age, yr } \\
\hline$<60$ & 115 & 45 & 70 & $>0.999$ \\
\hline$\geq 60$ & 24 & 9 & 15 & \\
\hline \multicolumn{5}{|l|}{ Sex } \\
\hline Male & 67 & 29 & 38 & 0.384 \\
\hline Female & 72 & 25 & 47 & \\
\hline \multicolumn{5}{|l|}{ ECOG } \\
\hline $\mathrm{o} / 1$ & 136 & 52 & 84 & 0.560 \\
\hline$\geq 2$ & 3 & 2 & 1 & \\
\hline \multicolumn{5}{|l|}{ Stage } \\
\hline II & 17 & 5 & 12 & 0.440 \\
\hline III/IV & 122 & 49 & 73 & \\
\hline \multicolumn{5}{|c|}{ Lactate dehydrogenase } \\
\hline Normal & 120 & 42 & 78 & 0.024 \\
\hline Elevated & 19 & 12 & 7 & \\
\hline \multicolumn{5}{|c|}{ Hemoglobin, g/dL } \\
\hline$<12$ & 19 & 10 & 9 & 0.211 \\
\hline$\geq 12$ & 120 & 44 & 76 & \\
\hline \multicolumn{5}{|l|}{ No. of nodal sites } \\
\hline$<5$ & 45 & 12 & 33 & 0.062 \\
\hline$\geq 5$ & 94 & 42 & 52 & \\
\hline \multicolumn{5}{|c|}{ Bone marrow involvement } \\
\hline Absence & 63 & 19 & 44 & 0.080 \\
\hline Presence & 76 & 35 & 41 & \\
\hline \multicolumn{5}{|l|}{ Grade } \\
\hline $1 / 2$ & $88 / 24$ & $26 / 11$ & $62 / 13$ & 0.007 \\
\hline 3 & 27 & 17 & 10 & \\
\hline \multicolumn{5}{|l|}{ FLIPI risk } \\
\hline Low & 36 & 10 & 26 & 0.115 \\
\hline Intermediate & 66 & 25 & 41 & \\
\hline High & 37 & 19 & 18 & \\
\hline \multicolumn{5}{|l|}{ HBsAg } \\
\hline Negative & 130 & 51 & 79 & $>0.999$ \\
\hline Positive & 9 & 3 & 6 & \\
\hline \multicolumn{5}{|l|}{$\mathrm{HBcAb}$} \\
\hline Negative & 83 & 33 & 50 & 0.860 \\
\hline Positive & 56 & 21 & 35 & \\
\hline
\end{tabular}

R-CHOP, rituximab, cyclophosphamide, doxorubicin, vincristine, and prednisone; R-CVP, rituximab, cyclophosphamide, vincristine, and prednisone; ECOG, Eastern Cooperative Oncology Group; FLIPI, Follicular Lymphoma International Prognostic Index; HBsAg, hepatitis B surface antigen; $\mathrm{HBcAb}$, hepatitis $\mathrm{B}$ core antibody.

\section{RESULTS}

\section{Patients}

The median age at diagnosis of the 139 patients was 48 years (range, 26 to 76 ). Of these, 85 patients received R-CVP and 54 patients received R-CHOP as induction treatment at the physicians' discretion. The ages of patients receiving R-CVP and R-CHOP did not differ significantly (median, 47.5 years [range, 27 to 75 ] vs. median, 48.4 years [range, 26 to 76 ]). The comparison of baseline characteristics showed no difference between the two groups in terms of stage and FLIPI risk $(p=0.440$ and $p$ $=0.115$, respectively) (Table 1 ). However, the proportion of patients with grade 3 disease was significantly higher in the R-CHOP group (17/54, 31\%) than in the R-CVP group $(10 / 85,12 \%, p=0.007)$ (Table 1). Furthermore, elevated serum LDH was more frequent in the R-CHOP group than in the R-CVP group $(p=0.024$ ) (Table 1 ). The viral marker status for HBV did not differ between the groups; nine patients were positive for $\mathrm{HBsAg}$ and $56 \mathrm{pa}-$ tients were positive for anti-HBc (Table 1). An antiviral agent was prophylactically used for HBsAg-positive patients before commencement of the first cycle of R-CVP or R-CHOP chemotherapy (seven received entecavir, two received tenofovir). Patients continuously took the antiviral agent during and after rituximab maintenance. However, antiviral prophylaxis was not used in HBsAg-negative/anti-HBc-positive patients.

\section{Treatment and survival outcomes}

The median follow-up was 29 months (95\% confidence interval, 23 to 45). Most patients received six cycles of $\mathrm{R}-\mathrm{CVP}$ or R-CHOP and the complete response rate did not differ significantly between R-CHOP (50/54, 92.6\%) and R-CVP (77/85, 90.6\%) (Table 2). More than half the patients in the R-CVP group completed 12 cycles of rituximab maintenance whereas $39 \%$ of patients in the R-CHOP group completed 12 cycles. The 3-year PFS and OS of all patients were $82 \%$ and $98 \%$, respectively. The number of disease relapses during rituximab maintenance did not differ significantly between groups ( $p=0.798$ ) (Table 2). Therefore, the comparison of PFS showed no significant difference: the 3-year PFS rates for R-CVP and R-CHOP were $77 \%$ and 85\%, respectively $(p=0.567$ ) (Fig. 1A). Of 17 patients who relapsed during or after rituximab maintenance, 11 relapsed within 24 
Table 2. Treatment and outcome

\begin{tabular}{|c|c|c|c|}
\hline Variable & $\mathrm{R}-\mathrm{CHOP}(\mathrm{n}=54)$ & $\mathrm{R}-\mathrm{CVP}(\mathrm{n}=85)$ & $p$ value \\
\hline \multicolumn{4}{|c|}{ No. of cycles of R-CVP or R-CHOP } \\
\hline 6 & 53 & 80 & 0.308 \\
\hline Other & 1 & 5 & \\
\hline \multicolumn{4}{|l|}{ Response } \\
\hline Complete response & 50 & 77 & 0.766 \\
\hline Partial response & 4 & 8 & \\
\hline \multicolumn{4}{|c|}{ No. of cycles of rituximab maintenance } \\
\hline 12 cycles & 21 & 44 & 0.164 \\
\hline$<12$ cycles & 33 & 41 & \\
\hline \multicolumn{4}{|l|}{ Relapse } \\
\hline No & 48 & 74 & 0.798 \\
\hline Yes & 6 & 11 & \\
\hline Hepatitis B virus reactivation & 2 & 3 & $>0.999$ \\
\hline $\mathrm{HBsAg}(+)$ & o & o & \\
\hline $\operatorname{HBsAg}(-) / \mathrm{HBcAb}(+)$ & 2 & 3 & \\
\hline $\operatorname{HBsAg}(-) / \mathrm{HBcAb}(-)$ & 0 & o & \\
\hline \multicolumn{4}{|l|}{ Survival status } \\
\hline Alive & 52 & 84 & 0.560 \\
\hline Dead & 2 & 1 & \\
\hline \multicolumn{4}{|l|}{ Cause of death } \\
\hline Disease-related & 1 & 0 & \\
\hline Nondisease-related & 1 & 1 & \\
\hline
\end{tabular}

R-CHOP, rituximab, cyclophosphamide, doxorubicin, vincristine, and prednisone; R-CVP, rituximab, cyclophosphamide, vincristine, and prednisone; HBsAg, hepatitis B surface antigen; HBcAb, hepatitis B core antibody.

months of diagnosis (R-CVP, $\mathrm{n}=7$; R-CHOP, $\mathrm{n}=4$ ) and six relapsed after more than 24 months $(\mathrm{R}-\mathrm{CVP}, \mathrm{n}=4$; $\mathrm{R}$-CHOP, $\mathrm{n}=2$ ). Autologous stem cell transplantation after salvage chemotherapy was performed in five patients who relapsed within 24 months. Of these, only one patient (male/61-year-old, stage III) died because of disease progression. The other 16 patients were alive at the time of analysis after they received salvage chemotherapy or radiotherapy or participated in a clinical trial. There were two cases of nondisease-related mortality; thus, two patients died of pneumonia and sepsis. Because the number of patients who died was relatively small, the OS did not differ between the two groups: the 3-year OS rates for R-CVP and R-CHOP were $97 \%$ and 98\%, respectively ( $p=0.124$ ) (Supplementary Fig. 1 ). A complete response to R-CVP or R-CHOP before rituximab maintenance was significantly associated with less frequent occurrence of disease relapse (11/127, 8.7\%) compared with a partial response $(6 / 12,50 \%)$. Thus, the PFS of patients with a complete response was significantly better than that of patients with a partial response (Fig. 1B). However, this was not related to OS (Supplementary Fig. 1).

\section{Risk factor analysis for PFS}

Because only three patients died, the risk factor analysis was done in terms of PFS. Sex, FL grade, elevation of serum LDH, and involvement of bone marrow were not significantly associated with worse PFS (Table 3). However, patients aged $\geq 60$ years showed a significantly shorter PFS and OS than patients younger than 60 years (Fig. 1C and Supplementary Fig. 1). PFS was significantly worse in the patients with high FLIPI risk (Fig. 1D). However, the FLIPI risk was not associated with OS 

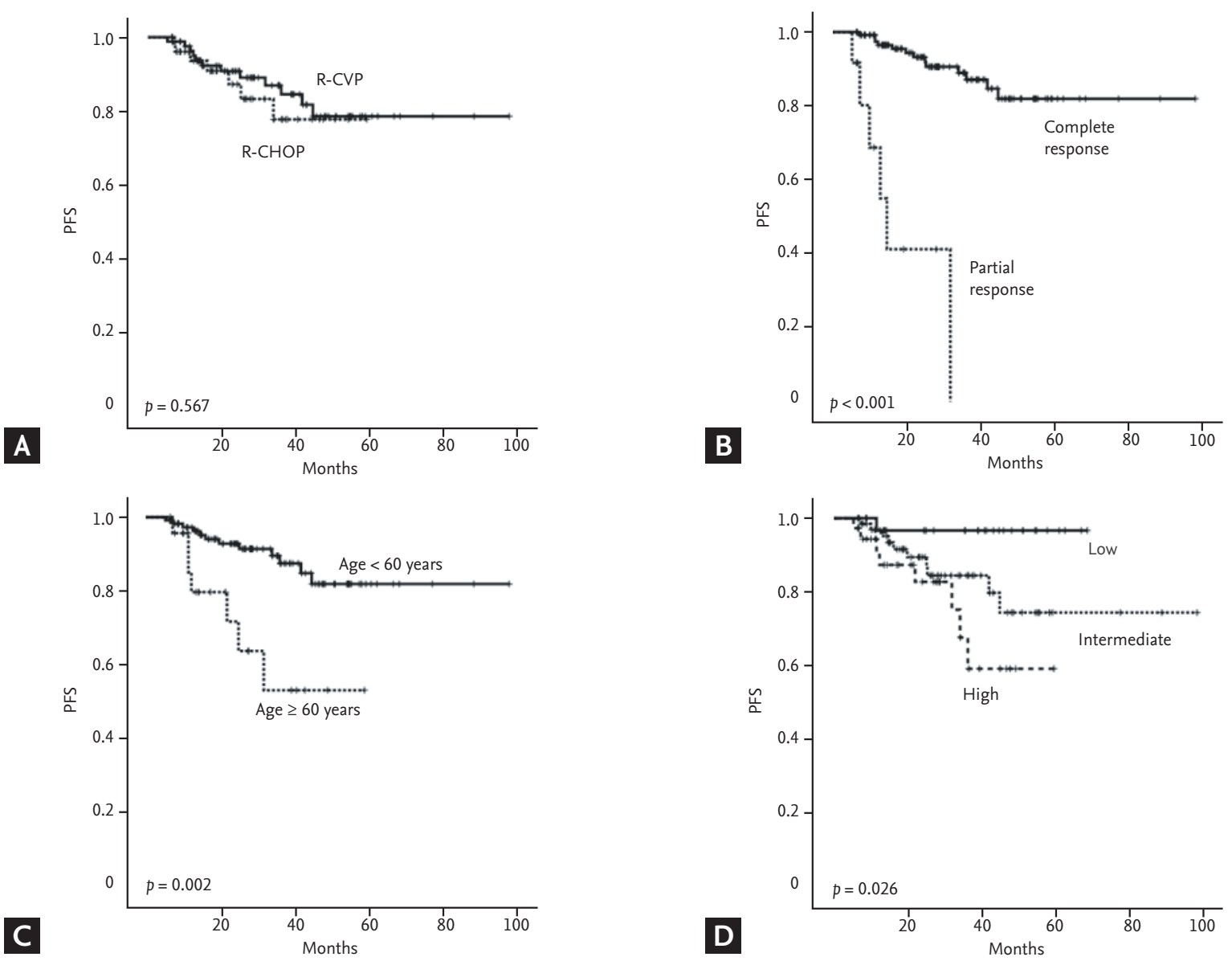

Fig. 1. (A) The comparison of progression-free survival (PFS) after R-CVP (rituximab, cyclophosphamide, vincristine, and prednisone) and R-CHOP (rituximab, cyclophosphamide, doxorubicin, vincristine, and prednisone). (B) Complete responders to R-CVP or R-CHOP show better PFS than partial responders. (C) The comparison of OS between patients aged < 60 years and $\geq 60$ years. (D) The comparison of OS according to the Follicular Lymphoma International Prognostic Index (FLIPI) risk group.

Table 3. Risk factor analysis for progression-free survival

\begin{tabular}{llll}
\hline Variable & HR & $95 \%$ CI & p value \\
\hline Age $\geq 60$ years & 4.025 & $1.570-10.318$ & 0.004 \\
Male sex & 0.768 & $0.311-1.894$ & 0.566 \\
ECOG $\geq 2$ & 3.951 & $0.521-29.962$ & 0.184 \\
Stage III/IV & 2.893 & $0.385-21.743$ & 0.302 \\
Elevated lactate dehydrogenase & 1.507 & $0.499-4.546$ & 0.467 \\
Hemoglobin $<12$ g/dL & 2.125 & $0.703-6.427$ & 0.182 \\
No. of nodal sites $\geq 5$ & 3.017 & $0.875-10.404$ & 0.080 \\
Bone marrow involvement & 1.412 & $0.555-3.587$ & 0.469 \\
Grade 3 & 1.197 & $0.397-3.613$ & 0.749 \\
FLIPI risk high & 2.486 & $1.254-4.931$ & 0.009 \\
\hline
\end{tabular}

HR, hazard ratio; CI, confidence interval; ECOG, Eastern Cooperative Oncology Group; FLIPI, Follicular Lymphoma International Prognostic Index. 

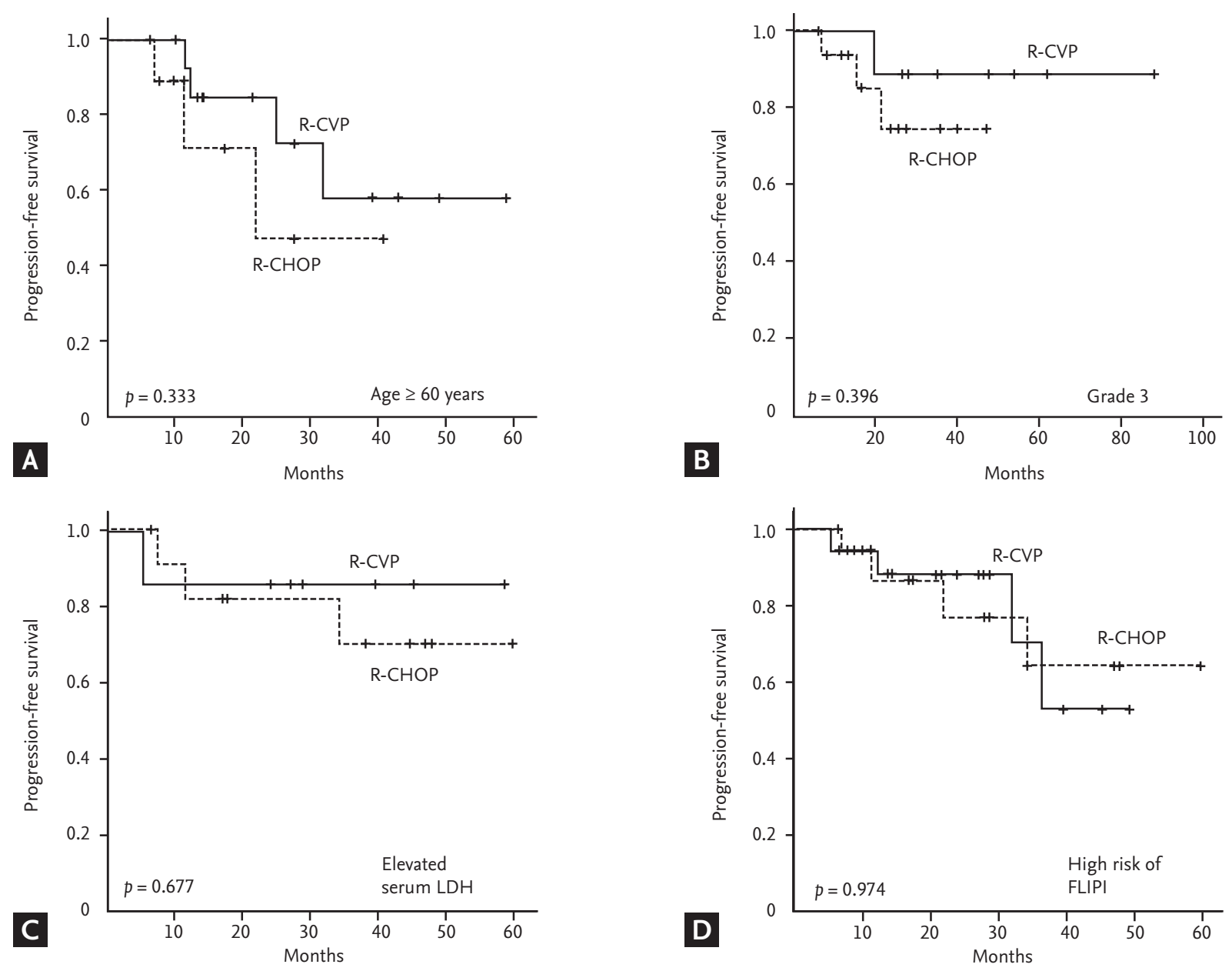

Fig. 2. Subgroup analysis with patients (A) age $\geq 60$ years, $(B)$ grade 3 disease, (C) elevated serum lactate dehydrogenase (LDH), and (D) high Follicular Lymphoma International Prognostic Index (FLIPI) risk shows no difference in progression-free survival between R-CVP (rituximab, cyclophosphamide, vincristine, and prednisone) and R-CHOP (rituximab, cyclophosphamide, doxorubicin, vincristine, and prednisone).

(Supplementary Fig. 1). The subgroup comparison of PFS in patients treated with R-CVP or R-CHOP showed no significant difference between the groups for age $\geq$ 60 years, grade 3 disease, elevated LDH, or high FLIPI risk (Fig. 2).

\section{Hepatitis $B$ virus reactivation}

There were five cases of $\mathrm{HBV}$ reactivation, all of which occurred in anti-HBc-positive patients. Three patients experienced HBV reactivation during or after rituximab maintenance therapy and two patients experienced HBV reactivation shortly after completing induction chemotherapy and before rituximab maintenance therapy (Table 4). All cases of HBV reactivation were identified during regular monitoring for HBV DNA in blood.
Patients did not show any symptoms of hepatitis, and their LFT values were normal, without conversion to HBsAg positive. These patients were able to continue to receive rituximab maintenance plus an antiviral agent after HBV reactivation was documented subsequent to induction chemotherapy. The clinical outcome of the treatment of HBV reactivation was successful in all cases, and all patients were alive in complete remission at the time of analysis.

\section{DISCUSSION}

This study analyzed the outcomes of FL patients who completed the planned cycles of induction chemother- 


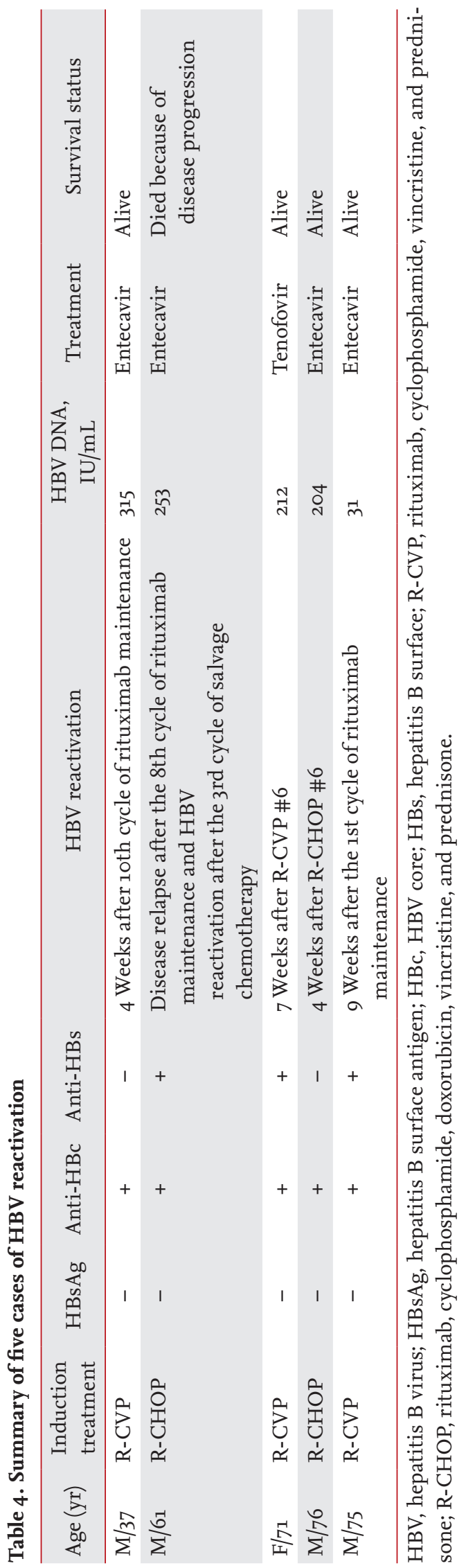

apy with R-CVP or R-CHOP, and received rituximab monotherapy every 2 months as a maintenance treatment. Because of the nature of this cross-sectional study, some patients were receiving the rituximab maintenance therapy at the time of analysis. Thus, approximately half the patients had completed their planned number of maintenance therapy cycles [12]. Despite this, the complete response rate, frequency of relapse, and survival outcome of the two induction therapies did not differ significantly (Table 2 and Fig. 1). However, these results should be interpreted carefully because it is possible that more patients with a high tumor burden or aggressive disease received R-CHOP rather than R-CVP, because physicians in general are concerned about the risk of treatment failure related to the use of the less-intensive treatment. Indeed, grade 3 disease and elevated serum LDH were more frequent in patients receiving R-CHOP, even though the other baseline characteristics did not differ significantly between the R-CVP and R-CHOP groups (Table 1). Nevertheless, the subgroup analysis including the patients with elevated serum $\mathrm{LDH}$ and grade 3 of FL showed no difference between the two groups. Furthermore, the OS did not differ between the two groups (Supplementary Fig. 1). Considering that this study included only patients who responded to induction treatment, because patients who were primarily refractory to R-CVP or R-CHOP were excluded from the analysis, these results could be interpreted as indicating that the survival outcomes of FL patients might not differ between R-CVP or R-CHOP provided they respond to the induction therapy, especially if they have a complete response.

Another concern about the treatment of FL patients is the benefit of rituximab maintenance. The proven benefit of rituximab maintenance is that it reduces the risk of relapse and delays the occurrence of relapse. Thus, the PRIMA study demonstrated a significant prolongation of PFS with rituximab maintenance therapy [22], although it failed to show that the treatment improved OS. This might be associated with the clinical course of FL, because FL patients can live longer than those with other aggressive lymphomas, even after repeated relapses. In our study, only one patient died because of disease progression and two patients died because of infections. The extremely low rate of disease-related mortality in this study might be associated with the relatively short- 
er median follow-up compared with previous studies. Thus, longer follow-up is required to determine the long-term outcomes of these patients and the occurrence of disease-related mortality. However, in this study, two patients died from infections during rituximab maintenance. It is well known that rituximab-induced B-cell depletion can impair humoral immunity, and because these patients were over 65 years old, their infections may have been related to prolonged B-cell depletion resulting from rituximab maintenance therapy. Thus, rituximab maintenance should be used cautiously in older patients who might be more vulnerable to infection.

This study also analyzed the association of HBV reactivation with rituximab maintenance in FL patients. There was no case of HBV reactivation in HBsAg-negative and anti-HBc-negative patients. Furthermore, the nine HBsAg-positive patients did not experience HBV reactivation because they received antiviral prophylaxis. However, five of the 56 anti-HBc-positive patients in our study experienced HBV reactivation. The presence of anti-HBc antibody can represent resolved HBV infection after prior exposure to HBV. Long-term depletion of B-cells, which occurs in rituximab maintenance, might increase the risk of $\mathrm{HBV}$ reactivation in anti-HBc-positive patients. Two of the cases of HBV reactivation were identified after the sixth cycle of R-CVP or R-CHOP whereas the other three occurred during rituximab maintenance. In this study, HBV reactivation was defined as conversion from undetectable to detectable serum levels of HBV DNA ( $\geq 10 \mathrm{IU} / \mathrm{mL}$ ). Therefore, all the cases of HBV reactivation were asymptomatic and were identified during regular monitoring of HBV DNA in blood, and all were successfully managed by subsequent antiviral treatment (Table 4). Thus, the regular monitoring of HBV DNA in blood could be a sufficient precaution for anti-HBc-positive FL patients who receive R-CVP or R-CHOP and rituximab maintenance. Although the number of patients with HBV reactivation was too small to allow a definite conclusion, four of the patients with HBV reactivation in our series were aged over 60 years (Table 4). Older age is a known risk factor for HBV reactivation after immunosuppressive or cytotoxic chemotherapy in B-cell lymphoma $[14,23]$. In a previous study including HBsAg-negative and anti-HBc-positive lymphoma patients receiving rituximab-containing chemotherapy without routine antiviral prophylaxis, patients aged over 70 years were reported to be particularly at risk for HBV reactivation [24]. Thus, older patients with FL should be carefully monitored using HBV DNA levels when on rituximab maintenance. The risk factor analysis for survival outcomes showed that age $\geq 60$ years was also significantly associated with decreased OS and PFS. This was related to the more frequent occurrence of relapse as well as nondisease-related mortality in older patients. Accordingly, the FLIPI risk was also associated with poor PFS because it includes age $\geq 60$ years as a risk factor. However, other parameters that have been traditionally considered unfavorable prognostic markers, including elevated serum LDH, disease stage, disease grade, and bone marrow involvement, were not related to survival outcomes in this study. Thus, age and FLIPI score should be considered the most important parameters when we plan to treat FL patients using rituximab-containing chemotherapy and rituximab maintenance.

In summary, our study showed that the survival outcomes of FL patients who received rituximab maintenance were similar once they had responded to R-CVP or R-CHOP. Furthermore, if HBV DNA titer in blood can be regularly monitored in anti-HBc-positive patients, rituximab-containing immunochemotherapy followed by rituximab maintenance can be safely used without resulting in serious HBV reactivation. However, there may be a risk of infection-related complications with rituximab-induced immune impairment, especially elderly patients. Thus, the probability of infectious complications should be considered in older, frail patients who might be vulnerable to infections.

\section{KEY MESSAGE}

1. Rituximab-containing immunochemotherapy followed by rituximab maintenance is an effective and safe treatment strategy for follicular lymphoma patients.

2. There is no significant difference in survival outcomes between R-CVP (rituximab, cyclophosphamide, vincristine, and prednisone) and R-CHOP (rituximab, cyclophosphamide, doxorubicin, vincristine, and prednisone) before rituximab maintenance. 


\section{Conflict of interest}

No potential conflict of interest relevant to this article was reported.

\section{Acknowledgments}

This study was supported by the Basic Science Research Program through the National Research Foundation of Korea (NRF) funded by the Ministry of Education, Science and Technology (NRF-2017R1A2B4005136).

\section{REFERENCES}

1. Morton LM, Wang SS, Devesa SS, Hartge P, Weisenburger DD, Linet MS. Lymphoma incidence patterns by WHO subtype in the United States, 1992-2001. Blood 2006;107:265-276.

2. Friedberg JW, Taylor MD, Cerhan JR, et al. Follicular lymphoma in the United States: first report of the national LymphoCare study. J Clin Oncol 2009;27:1202-1208.

3. Yoo KH, Lee H, Suh C; CISL. Lymphoma epidemiology in Korea and the real clinical field including the Consortium for Improving Survival of Lymphoma (CISL) trial. Int J Hematol 2018;107:395-404.

4. Cho SH, Suh C, Do YR, et al. Clinical features and survival of patients with follicular lymphoma in Korea. Clin Lymphoma Myeloma Leuk 2016;16:197-202.

5. Fisher RI, LeBlanc M, Press OW, Maloney DG, Unger JM, Miller TP. New treatment options have changed the survival of patients with follicular lymphoma. J Clin Oncol 2005;23:8447-8452.

6. Vidal L, Gafter-Gvili A, Salles G, et al. Rituximab maintenance for the treatment of patients with follicular lymphoma: an updated systematic review and meta-analysis of randomized trials. J Natl Cancer Inst 2011;103:17991806.

7. Luminari S, Ferrari A, Manni M, et al. Long-term results of the FOLLo5 trial comparing R-CVP versus R-CHOP versus R-FM for the initial treatment of patients with advanced-stage symptomatic follicular lymphoma. J Clin Oncol 2018;36:689-696.

8. Zhang L, Ghielmini M, Cheson BD, Ujjani C. Pros and cons of rituximab maintenance in follicular lymphoma. Cancer Treat Rev 2017;58:34-40.

9. Seto WK, Chan TS, Hwang YY, et al. Hepatitis B reactivation in patients with previous hepatitis B virus exposure undergoing rituximab-containing chemotherapy for lymphoma: a prospective study. J Clin Oncol 2014;32:37363743 .

10. Kim SJ, Hsu C, Song YQ, et al. Hepatitis B virus reactivation in B-cell lymphoma patients treated with rituximab: analysis from the Asia Lymphoma Study Group. Eur J Cancer 2013;49:3486-3496.

11. van der Kolk LE, Baars JW, Prins MH, van Oers MH. Rituximab treatment results in impaired secondary humoral immune responsiveness. Blood 2002;100:2257-2259.

12. Evens AM, Jovanovic BD, Su YC, et al. Rituximab-associated hepatitis $\mathrm{B}$ virus (HBV) reactivation in lymphoproliferative diseases: meta-analysis and examination of FDA safety reports. Ann Oncol 2011;22:1170-1180.

13. Liu CJ, Chen PJ, Chen DS, Kao JH. Hepatitis B virus reactivation in patients receiving cancer chemotherapy: natural history, pathogenesis, and management. Hepatol Int 2013;7:316-326.

14. Hayashi $\mathrm{K}$, Ishigami M, Ishizu $\mathrm{Y}$, et al. Clinical characteristics and molecular analysis of hepatitis $\mathrm{B}$ virus reactivation in hepatitis B surface antigen-negative patients during or after immunosuppressive or cytotoxic chemotherapy. J Gastroenterol 2016;51:1081-1089.

15. Dervite I, Hober D, Morel P. Acute hepatitis B in a patient with antibodies to hepatitis B surface antigen who was receiving rituximab. N Engl J Med 2001;344:68-69.

16. Dong HJ, Ni LN, Sheng GF, Song HL, Xu JZ, Ling Y. Risk of hepatitis B virus (HBV) reactivation in non-Hodgkin lymphoma patients receiving rituximab-chemotherapy: a meta-analysis. J Clin Virol 2013;57:209-214.

17. Barr PM, Li H, Burack WR, et al. R-CHOP, radioimmunotherapy, and maintenance rituximab in untreated follicular lymphoma (SWOG So801): a single-arm, phase 2, multicentre study. Lancet Haematol 2018;5:e102-e108.

18. Bech RS, Nielsen KL, Larsen TS, et al. Real world data on rituximab maintenance therapy after frontline immunochemotherapy in grade 1-3a follicular lymphoma. Br J Haematol 2018;182:297-301.

19. European Association for the Study of the Liver. EASL clinical practice guidelines: management of chronic hepatitis B virus infection. J Hepatol 2012;57:167-185.

20. Solal-Celigny P, Roy P, Colombat P, et al. Follicular lymphoma international prognostic index. Blood 2004;104:12581265 .

21. Cheson BD, Fisher RI, Barrington SF, et al. Recommendations for initial evaluation, staging, and response as- 
sessment of Hodgkin and non-Hodgkin lymphoma: the Lugano classification. J Clin Oncol 2014;32:3059-3068.

22. Salles G, Seymour JF, Offner F, et al. Rituximab maintenance for 2 years in patients with high tumour burden follicular lymphoma responding to rituximab plus chemotherapy (PRIMA): a phase 3, randomised controlled trial. Lancet 2011;377:42-51.

23. Kusumoto S, Tanaka Y, Suzuki R, et al. Monitoring of hepatitis B virus (HBV) DNA and risk of HBV reactiva- tion in B-cell lymphoma: a prospective observational study. Clin Infect Dis 2015;61:719-729.

24. Koo YX, Tay M, Teh YE, et al. Risk of hepatitis B virus (HBV) reactivation in hepatitis B surface antigen negative/hepatitis B core antibody positive patients receiving rituximab-containing combination chemotherapy without routine antiviral prophylaxis. Ann Hematol 2011;90:1219-1223. 

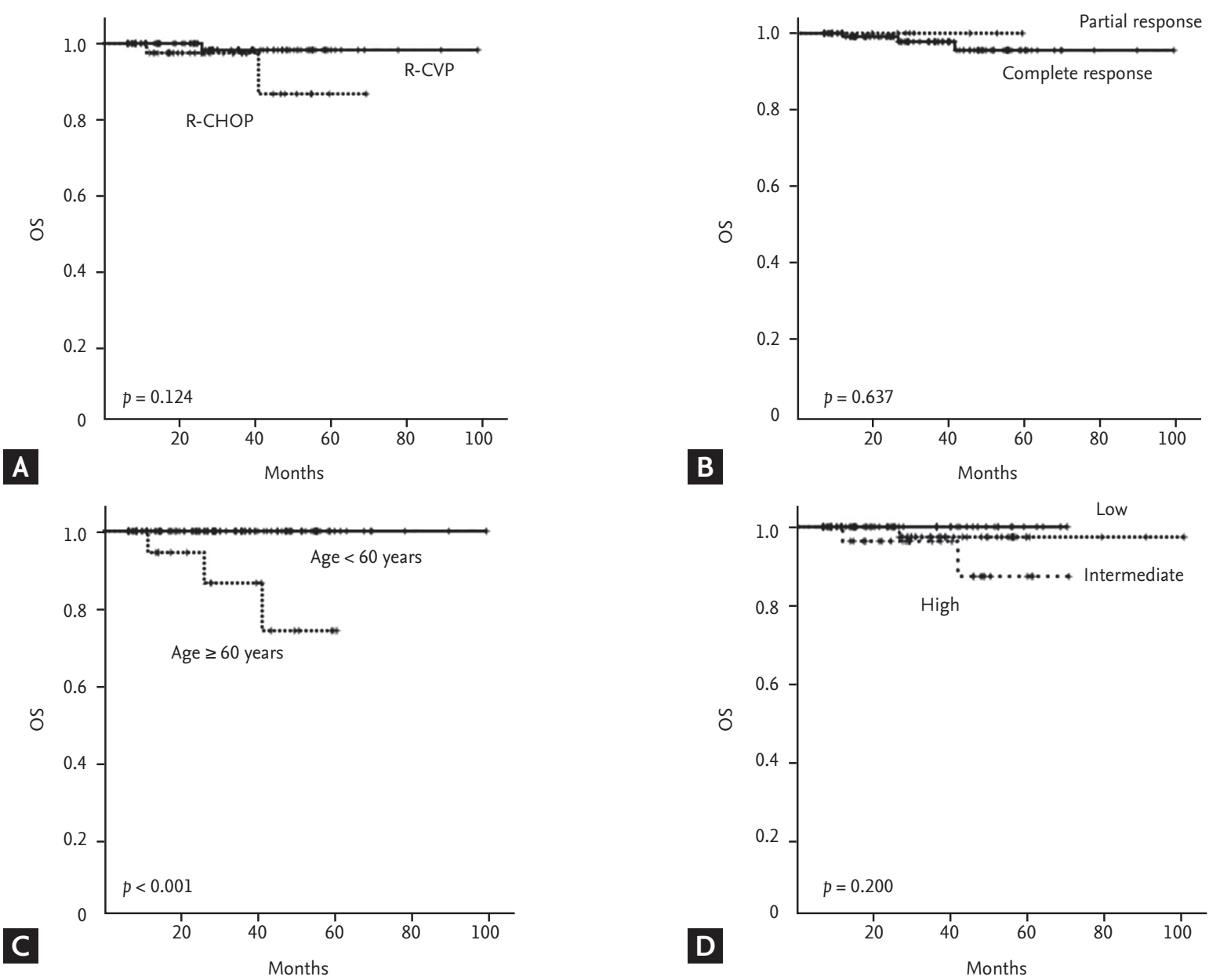

B

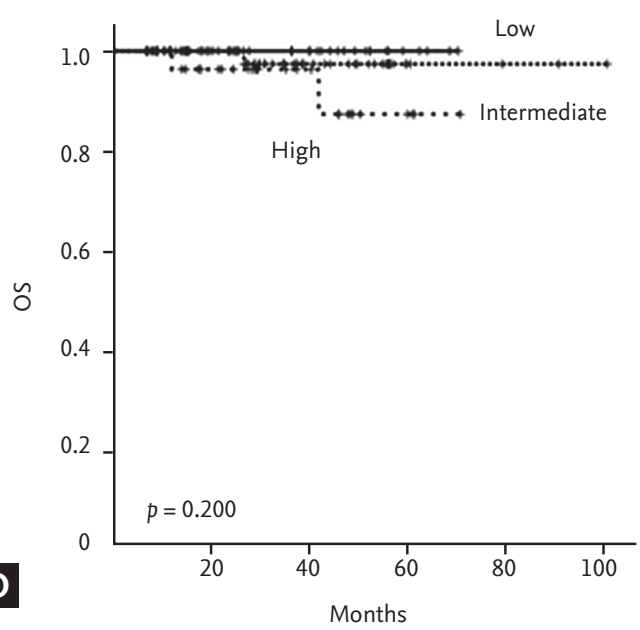

Supplementary Figure 1. (A) The comparison of overall survival (OS) after R-CVP (rituximab, cyclophosphamide, vincristine, and prednisone) and R-CHOP (rituximab, cyclophosphamide, doxorubicin, vincristine, and prednisone). (B) Complete and partial responders to R-CVP or R-CHOP show no significant difference in OS. (C) The comparison of OS between patients aged $<60$ and $\geq 60$ years. (D) The comparison of OS according to the Follicular Lymphoma International Prognostic Index (FLIPI) risk group. 